PROCEEDINGS OF THE

AMERICAN MATHEMATICAL SOCIETY

Volume 128, Number 3, Pages 727-734

$\mathrm{S}$ 0002-9939(99)05420-9

Article electronically published on September 9, 1999

\title{
ON AN ELEMENTARY APPROACH TO THE FRACTIONAL HARDY INEQUALITY
}

\author{
NATAN KRUGLJAK, LECH MALIGRANDA, AND LARS ERIK PERSSON
}

(Communicated by Frederick W. Gehring)

\begin{abstract}
Let $H$ be the usual Hardy operator, i.e., $H u(t)=\frac{1}{t} \int_{0}^{t} u(s) d s$. We prove that the operator $K=I-H$ is bounded and has a bounded inverse on the weighted spaces $L_{p}\left(t^{-\alpha}, d t / t\right)$ for $\alpha>-1$ and $\alpha \neq 0$. Moreover, by using these inequalities we derive a somewhat generalized form of some well-known fractional Hardy type inequalities and also of a result due to Bennett-DeVoreSharpley, where the usual Lorentz $L_{p, q}$ norm is replaced by an equivalent expression. Examples show that the restrictions in the theorems are essential.
\end{abstract}

\section{INTRODUCTION}

Let $u=u(t)$ denote a locally integrable function on $(0, \infty)$. First we note that if $1 \leq p \leq \infty$ and $\alpha>-1$, then, according to the Minkowski and Hardy inequalities,

$$
\left(\int_{0}^{\infty}\left|\frac{u(t)-\frac{1}{t} \int_{0}^{t} u(s) d s}{t^{\alpha}}\right|^{p} \frac{d t}{t}\right)^{1 / p} \leq\left(1+\frac{1}{\alpha+1}\right)\left(\int_{0}^{\infty}\left|\frac{u(t)}{t^{\alpha}}\right|^{p} \frac{d t}{t}\right)^{1 / p}
$$

Of course a reversed inequality of the type

$$
\left(\int_{0}^{\infty}\left|\frac{u(t)}{t^{\alpha}}\right|^{p} \frac{d t}{t}\right)^{1 / p} \leq C\left(\int_{0}^{\infty}\left|\frac{u(t)-\frac{1}{t} \int_{0}^{t} u(s) d s}{t^{\alpha}}\right|^{p} \frac{d t}{t}\right)^{1 / p}
$$

cannot hold in general (for example it does not hold for the function $u \equiv$ constant; see also Remark 1). One main purpose of this paper is to prove that (1.2) in fact holds for every $\alpha \neq 0$ if we impose some (relatively small) restrictions on the functions $u$. We formulate and prove this result in Section 2. In Section 3 we use this result to prove a generalized form of a well-known fractional Hardy type inequality (see [5], [13] and cf. also [4], [6] and the references given there). Moreover, we point out the fact that our result may also be considered as a generalized form of a result due to Bennett-DeVore-Sharpley, where the usual Lorentz $L_{p, q}$ norm is replaced by an equivalent expression (see [2] and also [3, p. 384]).

In order not to disturb our discussions later on we give some necessary notation and other preliminaries. By $u=u(x)$ we denote a locally integrable function on

Received by the editors April 15, 1998.

1991 Mathematics Subject Classification. Primary 26D15; Secondary 46E30.

Key words and phrases. Inequalities, Hardy inequality, Grisvard inequality, Lorentz spaces. 
$(0, \infty)$, i.e., $\int_{a}^{b}|u(t)| d t<\infty$ for $0<a<b<\infty$. Moreover, by the integrals $\int_{0}^{t} u(s) d s, t>0$, and $\int_{0}^{\infty} u(s) d s$ we mean

$$
\int_{0}^{t} u(s) d s=\lim _{\varepsilon \rightarrow 0+} \int_{\varepsilon}^{t} u(s) d s, \quad \operatorname{resp} . \int_{0}^{\infty} u(s) d s=\lim _{t \rightarrow \infty} \int_{0}^{t} u(s) d s .
$$

Also the following simple observation has helped guide our investigations:

Lemma 1. Let $u \in L_{p}\left(t^{-\alpha}, d t / t\right), 1 \leq p \leq \infty$. Then

(a) $\int_{0}^{t} u(s) d s$ exists if $\alpha>-1$,

(b) $\lim _{t \rightarrow 0} \frac{1}{t} \int_{0}^{t} u(s) d s=0$ if $\alpha>0$, and

(c) $\lim _{t \rightarrow \infty} \frac{1}{t} \int_{0}^{t} u(s) d s=0$ if $-1<\alpha<0$.

The proof consists only of an obvious application of the Hölder inequality.

\section{The MAIN RESUlt}

Our main result reads:

Theorem 1. Let $1 \leq p \leq \infty, \alpha \in \mathbb{R} \backslash\{0\}$ and assume that $\int_{0}^{t} u(s) d s$ exists. Then

$$
\left(\int_{0}^{\infty}\left|\frac{u(t)}{t^{\alpha}}\right|^{p} \frac{d t}{t}\right)^{1 / p} \leq C(\alpha)\left(\int_{0}^{\infty}\left|\frac{u(t)-\frac{1}{t} \int_{0}^{t} u(s) d s}{t^{\alpha}}\right|^{p} \frac{d t}{t}\right)^{1 / p}
$$

where $C(\alpha)=1+1 /|\alpha|$, in each of the following cases:

a) $a>0$ and $\lim _{t \rightarrow 0} \frac{1}{t} \int_{0}^{t} u(s) d s=0$,

b) $\alpha<0$ and $\lim _{t \rightarrow \infty} \frac{1}{t} \int_{0}^{t} u(s) d s=0$.

Remark 1. In particular, Theorem 1 implies that the inequality (2.1) holds for all $\alpha \in \mathbb{R} \backslash\{0\}$ and any locally integrable function $u$ with compact support in $(0, \infty)$.

Remark 2. The inequality (2.1) does not hold for $\alpha=0$ even if we assume that $\int_{0}^{t} u(s) d s$ exists and $\lim _{t \rightarrow 0} \frac{1}{t} \int_{0}^{t} u(s) d s=0$ or $\lim _{t \rightarrow \infty} \frac{1}{t} \int_{0}^{t} u(s) d s=0$. For $p<\infty$ we insert $u_{a}(t)=\chi_{[1, a]}(t), 1<a<\infty$, into (2.1) and let $a \rightarrow \infty$ to obtain a suitable counterexample. For $p=\infty$ we obtain a counterexample by inserting the functions $u_{\varepsilon}(t)=t^{\varepsilon} \chi_{(0,1)}(t)+\chi_{[1, \infty)}(t)$ into $(2.1)$ and letting $\varepsilon \rightarrow 0$.

Remark 3. If, in addition to the assumptions in Theorem 1, we assume that $\alpha>-1$, then, according to (1.1), we see that $(2.1)$ can be replaced by the equivalence ${ }^{1}$

$$
\left(\int_{0}^{\infty}\left|\frac{u(t)}{t^{a}}\right|^{p} \frac{d t}{t}\right)^{1 / p} \approx\left(\int_{0}^{\infty}\left|\frac{u(t)-\frac{1}{t} \int_{0}^{t} u(s) d s}{t^{\alpha}}\right|^{p} \frac{d t}{t}\right)^{1 / p} .
$$

In view of Lemma 1 this means in particular that the expression on the right hand side of (2.2) gives an equivalent norm of $L_{p}\left(t^{-\alpha}, d t / t\right)$ for $\alpha \in(-1,0)$ or $\alpha \in(0, \infty)$.

Proof of Theorem 1. Let $v=(I-H) u$, i.e.,

$$
v(t)=u(t)-\frac{1}{t} \int_{0}^{t} u(s) d s
$$

\footnotetext{
${ }^{1}$ Here the symbol $\approx$ means that the constants of equivalence are independent of $u$ for a fixed $\alpha$ and $p$.
} 
Then, for $0<t_{0}<t_{1}<\infty$ we find, by integrating by parts,

$$
\begin{aligned}
\int_{t_{0}}^{t_{1}} \frac{v(s)}{s} d s & =\int_{t_{0}}^{t_{1}} \frac{u(s)}{s} d s-\int_{t_{0}}^{t_{1}}\left(\frac{1}{s^{2}} \int_{0}^{s} u(\tau) d \tau\right) d s \\
& =\int_{t_{0}}^{t_{1}} \frac{u(s)}{s} d s+\left[\frac{1}{s} \int_{0}^{s} u(\tau) d \tau\right]_{t_{0}}^{t_{1}}-\int_{t_{0}}^{t_{1}} \frac{u(s)}{s} d s .
\end{aligned}
$$

Therefore, by only using the condition that $\int_{0}^{t} u(s) d s$ exists we have proved that

$$
\int_{t_{0}}^{t_{1}} \frac{v(s)}{s} d s=\frac{1}{t_{1}} \int_{0}^{t_{1}} u(s) d s-\frac{1}{t_{0}} \int_{0}^{t_{0}} u(s) d s .
$$

Now, by putting $t_{1}=t$ in (2.4) and letting $t_{0} \rightarrow 0$, we obtain that for case a) it yields that

$$
\int_{0}^{t} \frac{v(s)}{s} d s=\frac{1}{t} \int_{0}^{t} u(s) d s
$$

By substituting this expression into (2.3) we obtain the following expression (for the inverse of the operator $K=I-H)$ :

$$
u(t)=v(t)+\int_{0}^{t} \frac{v(s)}{s} d s
$$

Analogously, by putting $t_{0}=t$ and letting $t_{1} \rightarrow \infty$ in (2.4) we find that for case b) it yields that

$$
\int_{t}^{\infty} \frac{v(s)}{s} d s=-\frac{1}{t} \int_{0}^{t} u(s) d s
$$

By substituting (2.7) into (2.3) we get the following formula (for the inverse of the operator $K=I-H)$ :

$$
u(t)=v(t)-\int_{t}^{\infty} \frac{v(s)}{s} d s .
$$

Now, the estimate (2.1) follows by using (2.6), (2.8) and the Hardy and Minkowski inequalities in the following ways: In case a)

$$
\begin{aligned}
\left(\int_{0}^{\infty}\left|\frac{u(t)}{t^{\alpha}}\right|^{p} \frac{d t}{t}\right)^{1 / p} & \leq\left(\int_{0}^{\infty}\left|\frac{v(t)}{t^{\alpha}}\right|^{p} \frac{d t}{t}\right)^{1 / p}+\left(\int_{0}^{\infty}\left|t^{-\alpha} \int_{0}^{t} \frac{v(s)}{s} d s\right|^{p} \frac{d t}{t}\right)^{1 / p} \\
& \leq\left(1+\frac{1}{\alpha}\right)\left(\int_{0}^{\infty}\left|\frac{v(t)}{t^{\alpha}}\right|^{p} \frac{d t}{t}\right)^{1 / p} \\
& =\left(1+\frac{1}{\alpha}\right)\left(\int_{0}^{\infty}\left|\frac{u(t)-\frac{1}{t} \int_{0}^{t} u(s) d s}{t^{\alpha}}\right|^{p} \frac{d t}{t}\right)^{1 / p}
\end{aligned}
$$


and in case b)

$$
\begin{aligned}
\left(\int_{0}^{\infty}\left|\frac{u(t)}{t^{\alpha}}\right|^{p} \frac{d t}{t}\right)^{1 / p} & \leq\left(\int_{0}^{\infty}\left|\frac{v(t)}{t^{\alpha}}\right|^{p} \frac{d t}{t}\right)^{1 / p}+\left(\int_{0}^{\infty}\left|t^{-\alpha} \int_{t}^{\infty} \frac{v(s)}{s} d s\right|^{p} \frac{d t}{t}\right)^{1 / p} \\
& \leq\left(1+\frac{1}{|\alpha|}\right)\left(\int_{0}^{\infty}\left|\frac{v(t)}{t^{\alpha}}\right|^{p} \frac{d t}{t}\right)^{1 / p} \\
& =\left(1+\frac{1}{|\alpha|}\right)\left(\int_{0}^{\infty}\left|\frac{u(t)-\frac{1}{t} \int_{0}^{t} u(s) d s}{t^{\alpha}}\right|^{p} \frac{d t}{t}\right)^{1 / p} .
\end{aligned}
$$

The proof is complete.

Remark 4. The inequality (2.1) does not hold for $0<p<1$ with any positive constant $C$. In order to see this we do the following: first we take $v(t)=\chi_{[1,1+\varepsilon]}(t)$, $\varepsilon>0$. After that we calculate a corresponding $u(t)=u_{\varepsilon}(t)$, from $(2.6)$ for the case $\alpha>0$,

$$
u_{\varepsilon}(t)=(1+\ln t) \chi_{[1,1+\varepsilon]}(t)+\ln (1+\varepsilon) \chi_{[1+\varepsilon, \infty)}(t),
$$

and from (2.8) for the case $\alpha<0$,

$$
u_{\varepsilon}(t)=-\ln (1+\varepsilon) \chi_{(0,1)}(t)+(1+\ln t-\ln (1+\varepsilon)) \chi_{[1,1+\varepsilon]}(t) .
$$

By inserting these functions $u(t)=u_{\varepsilon}(t)$ into (2.1) and letting $\varepsilon \rightarrow 0$ we see that the constant $C(\alpha)$ tends to $\infty$.

Remark 5. According to Theorem 1 we see that the operator $K=I-H$ is bounded and has a bounded inverse for all $\alpha$ such that $-1<\alpha<0$ or $\alpha>0$. On the other hand, in view of (1.1) and the counterexample to (1.2) for $\alpha=0$ we see that for $\alpha=0$ the operator $K=I-H$ is bounded in $L_{p}\left(t^{-\alpha}, d t / t\right)$ but it does not have bounded inverse. From an interpolation point of view it can be confusing that we have an inequality with weights $t^{\alpha}$ which hold both for $\alpha>0$ and for $\alpha<0$ but does not hold for $\alpha=0$. The explanation of this phenomenon can be understood from our proof above where we have seen that the inverse operator of $K=I-H$ is different for $\alpha>0$ and for $\alpha<0$ (see (2.6) and (2.8)), i.e,

$$
\begin{array}{ll}
\left(K^{-1} v\right)(t)=v(t)+\int_{0}^{t} \frac{v(s)}{s} d s & (\alpha>0), \\
\left(K^{-1} v\right)(t)=v(t)-\int_{t}^{\infty} \frac{v(s)}{s} d s & (-1<\alpha<0)
\end{array}
$$

and to interpolate we need to have exactly one operator (more about this type of phenomenon can be found in [7]).

\section{A FRACTIONAL HARDY INEQUALity AND FINAL REMARKS}

It is known that if $u \in C_{0}^{\infty}(0, \infty), 1<p<\infty, 0<\theta<1, \theta \neq 1 / p$, then

$$
\left(\int_{0}^{\infty}\left|\frac{u(t)}{t^{\theta}}\right|^{p} d t\right)^{1 / p} \leq C\left(\int_{0}^{\infty} \int_{0}^{\infty} \frac{|u(t)-u(s)|^{p}}{|t-s|^{\theta p+1}} d t d s\right)^{1 / p} .
$$

Sometimes this inequality is called the Grisvard inequality (see [5]) but in fact this inequality was known even before e.g. in the works of Aronszajn-Smith (Technical Report from 1961, cf. [1]), Lions-Magenes [10] and Yakovlev [13]. The inequality (3.1) can be interpreted as a "fractional order Hardy inequality" (where the usual 
Hardy inequality corresponds to the endpoint case $\theta=1$ ). Some other generalizations of (3.1) have recently been obtained in [4] and [6]. Here we state another generalization as a consequence of our Theorem 1.

Theorem 2. Let $1 \leq p \leq \infty, \theta \in \mathbb{R}$ and $u$ be a locally integrable function on $(0, \infty)$. Then the equality

$$
\left(\int_{0}^{\infty}\left|\frac{u(t)}{t^{\theta}}\right|^{p} d t\right)^{1 / p} \leq C(\theta, p)\left(\int_{0}^{\infty} \int_{0}^{\infty} \frac{|u(t)-u(s)|^{p}}{|t-s|^{\theta p+1}} d t d s\right)^{1 / p},
$$

where $C(\theta, p)=2^{-1 / p}(1+1 /|\theta-1 / p|)$, holds in each of the following cases:

a) $1 / p<\theta<1$ and $\lim _{t \rightarrow 0} \frac{1}{t} \int_{0}^{t} u(s) d s=0$,

b) $0<\theta<1 / p$ and $\lim _{t \rightarrow \infty} \frac{1}{t} \int_{0}^{t} u(s) d s=0$.

Remark 6 . The restrictions of the parameter $\theta$ in Theorem 2 are essential. In fact,

a) if either $\theta \geq 1$ or $\theta \leq 0$, then the integral on the right hand side of (3.2) diverges e.g. for each function $u \in C_{0}^{\infty}(0, \infty)(u \neq 0)$;

b) if $\theta=1 / p, 1<p<\infty$, then, by inserting the functions

$$
u_{\varepsilon}(t)=\frac{t-\varepsilon}{\varepsilon} \chi_{[\varepsilon, 2 \varepsilon)}(t)+\chi_{[2 \varepsilon, 1 / 2)}(t)+2(1-t) \chi_{[1 / 2,1)}(t)
$$

into (3.2) and letting $\varepsilon \rightarrow 0$, we find (after some straightforward calculations) that the constant $C(\theta, p) \rightarrow \infty$.

Proof of Theorem 2. First let $1 \leq p<\infty$ and put $\alpha=\theta-1 / p$. We note that, by Hölder's inequality,

$$
\left|u(t)-\frac{1}{t} \int_{0}^{t} u(s) d s\right|^{p}=\left|\frac{1}{t} \int_{0}^{t}(u(t)-u(s)) d s\right|^{p} \leq \frac{1}{t} \int_{0}^{t}|u(t)-u(s)|^{p} d s .
$$

Therefore, since also $\theta \geq-1 / p$ so that $\alpha p+2 \geq 0$,

$$
\begin{aligned}
& \left(\int_{0}^{\infty}\left|\frac{u(t)-\frac{1}{t} \int_{0}^{t} u(s) d s}{t^{\theta}}\right|^{p} d t\right)^{1 / p}=\left(\int_{0}^{\infty}\left|\frac{u(t)-\frac{1}{t} \int_{0}^{t} u(s) d s}{t^{\alpha}}\right|^{p} \frac{d t}{t}\right)^{1 / p} \\
& \leq\left(\int_{0}^{\infty} \int_{0}^{t} \frac{|u(t)-u(s)|^{p}}{t^{\alpha p+2}} d s d t\right)^{1 / p} \leq\left(\int_{0}^{\infty} \int_{0}^{t} \frac{|u(t)-u(s)|^{p}}{|t-s|^{\alpha p+2}} d s d t\right)^{1 / p} \\
& \quad=\left(\frac{1}{2} \int_{0}^{\infty} \int_{0}^{\infty} \frac{|u(t)-u(s)|^{p}}{|t-s|^{\alpha p+2}} d s d t\right)^{1 / p}=\left(\frac{1}{2} \int_{0}^{\infty} \int_{0}^{\infty} \frac{|u(t)-u(s)|^{p}}{|t-s|^{\theta p+1}} d s d t\right)^{1 / p} .
\end{aligned}
$$

The proof now follows by using this estimate and Theorem 1 . The proof for the case $p=\infty$ consists only of some obvious modifications.

Remark 7. Recall that our inequality (2.1) does not hold for $0<p<1$ (see Remark 4). Therefore it is a surprising fact that, if $u$ and $q$ satisfy the assumptions in Theorem 2, then the inequality (3.1) in fact holds also for the case $0<p<1$; cf. [8, p. 19]. This fact can be seen by choosing numbers $q$ and $\lambda$ such that $\lambda q=\theta p$ and $1 \leq q<\infty$, by applying Theorem 2 with $q$ and $\lambda$ instead of $p$ and $\theta$, respectively, to the function $|u(t)|^{p / q}$ instead of $u(t)$ and using the elementary inequality

$$
\left.|| u(t)\right|^{p / q}-|u(s)|^{p / q}|\leq| u(t)-\left.u(s)\right|^{p / q} .
$$

Sometimes the formula (3.1) is written in a little different form in the literature (see e.g. [1], [10], [13]). In order to also cover such cases we reformulate our Theorem 2 in the following equivalent way. 
Theorem $2^{\prime}$. Let $1 \leq p \leq \infty, \theta \in \mathbb{R}$ and $u$ be a locally integrable function on $(0, \infty)$. Then the inequality

$$
\left(\int_{0}^{\infty}\left|\frac{u(t)-\bar{u}}{t^{\theta}}\right|^{p} d t\right)^{1 / p} \leq C(\theta, p)\left(\int_{0}^{\infty} \int_{0}^{\infty} \frac{|u(t)-u(s)|^{p}}{|t-s|^{\theta p+1}} d t d s\right)^{1 / p},
$$

where $C(\theta, p)=2^{-1 / p}(1+1 /|\theta-1 / p|)$, holds in each of the following cases:

a) $1 / p<\theta<1$ and $\lim _{t \rightarrow 0} \frac{1}{t} \int_{0}^{t} u(s) d s=\bar{u}$,

b) $0<\theta<1 / p$ and $\lim _{t \rightarrow \infty} \frac{1}{t} \int_{0}^{t} u(s) d s=\bar{u}$.

Now let $f$ denote a measurable function on the totally $\sigma$-finite measure space $(\Omega, \mu)$. Moreover, let $f^{*}$ denote the nonincreasing rearrangement of $f$ and

$$
f^{* *}(t)=\frac{1}{t} \int_{0}^{t} f^{*}(s) d s .
$$

In [2] Bennett-DeVore-Sharpley used the functional $f^{* *}-f^{*}$ in the definition of the "weak- $L^{\infty}$ " space and also proved an interpolation result in this connection (see also [3]). Moreover, it is proved in [3, Proposition 7.12] that if $f \in L_{p, q}, 1<p<\infty$, $1 \leq q \leq \infty$, and $f^{* *}(\infty)=\lim _{t \rightarrow \infty} f^{* *}(t)=0$, then

$$
\|f\|_{L_{p, q}}:=\left(\int_{0}^{\infty}\left(t^{1 / p} f^{*}(t)\right)^{q} \frac{d t}{t}\right)^{1 / q} \approx\left(\int_{0}^{\infty}\left(t^{1 / p}\left(f^{* *}(t)-f^{*}(t)\right)\right)^{q} \frac{d t}{t}\right)^{1 / q},
$$

with the usual (supremum) interpretation for the case $q=\infty$.

By using our Theorem 1 and the estimate (1.1) with $\alpha=-1 / p$ and $p$ replaced by $q$ to the function $u=f^{*}$ we obtain the following somewhat more precise information:

Proposition 1. Let $f^{*}$ and $f^{* *}$ be defined as above.

a) If $1<p<\infty, 1 \leq q \leq \infty$, then

$$
\left(\int_{0}^{\infty}\left(t^{1 / p}\left(f^{* *}(t)-f^{*}(t)\right)\right)^{q} \frac{d t}{t}\right)^{1 / q} \leq\left(1+\frac{p}{p-1}\right)\left(\int_{0}^{\infty}\left(t^{1 / p} f^{*}(t)\right)^{q} \frac{d t}{t}\right)^{1 / q} .
$$

b) If $0<p<\infty, 1 \leq q \leq \infty$, and $f^{* *}(\infty)=0$, then

$$
\left(\int_{0}^{\infty}\left(t^{1 / p} f^{*}(t)\right)^{q} \frac{d t}{t}\right)^{1 / q} \leq(p+1)\left(\int_{0}^{\infty}\left(t^{1 / p}\left(f^{* *}(t)-f^{*}(t)\right)\right)^{q} \frac{d t}{t}\right)^{1 / q} .
$$

Remark 8. Observe that the estimates in Proposition 1 imply the equivalence between $f$ in the Lorentz $L_{p, q}$ space and $f^{* *}-f^{*}$ in a weighted $L_{q^{-}}$-space. Moreover, it is well-known that $f$ and $f_{\#}(t):=\frac{1}{t} \int_{0}^{t} f(s) d s-f(t)$ are equivalent in Lorentz $L_{p q}$ spaces (see [11]).

Proposition 2. a) If $1<p<\infty, 0<q \leq \infty$, then

$$
\left\|f_{\#}\right\|_{L_{p q}} \leq C_{p, q}\|f\|_{L_{p q}} .
$$

b) If $1<p<\infty, 0<q \leq \infty$ and $\lim _{t \rightarrow \infty} \frac{1}{t} \int_{0}^{t} f(s) d s=0$, then

$$
\|f\|_{L_{p q}} \leq D_{p, q}\left\|f_{\#}\right\|_{L_{p q}} .
$$

This statement was proved by Milman and Sagher [11] by using interpolation techniques. Here we give a direct and completely different proof. 
Proof of Proposition 2. a) We note that

$$
\left|f_{\#}(t)\right| \leq \frac{1}{t} \int_{0}^{t}|f(s)| d s+|f(t)| \leq \frac{1}{t} \int_{0}^{t} f^{*}(s) d s+|f(t)|
$$

and it follows that

$$
\left(f_{\#}\right)^{*}(t) \leq \frac{2}{t} \int_{0}^{t / 2} f^{*}(s) d s+f^{*}(t / 2) .
$$

Thus the proof follows by using the Minkowski and Hardy inequalities.

b) Similarly as in the proof of Theorem 1 we find that

$$
f(t)=-f_{\#}(t)+\int_{t}^{\infty} f_{\#}(s) \frac{d s}{s} .
$$

We must now consider each of the cases $1 \leq q \leq p, 1<p \leq q$ and $0<q<1<p<$ $\infty$. In each case the proof follows by using the Minkowski and Hardy inequalities. In the second case we must also use Köthe duality and in the third case we must first do the following calculation:

$$
\begin{aligned}
\int_{t}^{\infty} f_{\#}(s) \frac{d s}{s} & =\int_{t}^{\infty} f_{\#}(s) \frac{1}{s} \chi_{[t, \infty)}(s) d s \leq \int_{0}^{\infty}\left(f_{\#}\right)^{*}(s)\left(\frac{1}{s} \chi_{[t, \infty)}\right)^{*}(s) d s \\
& =\int_{0}^{\infty}\left(f_{\#}\right)^{*}(s) \frac{1}{s+t} d s \leq \frac{1}{t} \int_{0}^{t}\left(f_{\#}\right)^{*}(s) d s+\int_{t}^{\infty}\left(f_{\#}\right)^{*}(s) \frac{d s}{s} .
\end{aligned}
$$

Remark 9. The estimate (1.1) together with our Theorem 1 obviously has some interpretations in techniques. For example, if $\alpha=-1 / 2$ and $p=2$ we obtain that

$$
\begin{aligned}
& \frac{1}{3}\left(\int_{0}^{\infty}\left(u(t)-\frac{1}{t} \int_{0}^{t} u(s) d s\right)^{2} d t\right)^{1 / 2} \\
& \quad \leq\left(\int_{0}^{\infty} u^{2}(t) d t\right)^{1 / 2} \leq 3\left(\int_{0}^{\infty}\left(u(t)-\frac{1}{t} \int_{0}^{t} u(s) d s\right)^{2} d t\right)^{1 / 2} .
\end{aligned}
$$

If, for example, $u=u(t)$ stands for a signal (or current) at the time $t$ and $v(t):=$ $u(t)-\frac{1}{t} \int_{0}^{t} u(s) d s$ is the "momentaneous noise of the signal", then (3.3) means that the energies of the signals $u(t)$ and $v(t)$ are comparable. For example if $u(t)=$ $\chi_{(0, a]}, a>0$, is the "unit step function", then we see that all energy of the signal $u(t)$ is concentrated on $(0, a]$ and all energy of the signal $v(t)$ on $[a, \infty)$, but the two energies can be compared in this good way.

\section{ACKNOWLEDGEMENTS}

The first author thanks Luleå University of Technology (Sweden) for financial support for his research stay in January 1998, which made this cooperation possible. The second author was partly supported by grant M-AA/MA 06857-306 of the Swedish Natural Science Research Council (NFR).

\section{Added IN PRoof (June 1999)}

After the acceptance of this paper we have found surprisingly elementary proofs of the following important complementary information to the results obtained in 
this paper:

1. (cf. Theorem 2) Let $1<p<\infty$ and $0<\theta<1$. Then, for every $u \in A C(0, \infty)$,

$$
\left(\int_{0}^{\infty} \int_{0}^{\infty} \frac{|u(t)-u(s)|^{p}}{|t-s|^{\theta p+1}} d t d s\right)^{1 / p} \leq C\left(\int_{0}^{\infty}\left|u^{\prime}(t)\right|^{p} t^{(1-\theta) p} d t\right)^{1 / p}
$$

2. (cf. Remark 9) If $u \in L_{2}=L_{2}(0, \infty)$, then we have in fact equality in (3.3), i.e.,

$$
\left(\int_{0}^{\infty}\left(u(t)-\frac{1}{t} \int_{0}^{t} u(s) d s\right)^{2} d t\right)^{1 / 2}=\left(\int_{0}^{\infty} u^{2}(t) d t\right)^{1 / 2},
$$

and even some more general weighted $L^{2}$ identities are true which can be found in: N. Kaiblinger, L. Maligranda and L. E. Persson, Norms in weighed $L^{2}$-spaces and Hardy operators, Research report 1999.

\section{REFERENCES}

[1] R. Adams, N. Aronszajn and K. T. Smith, Theory of Bessel potentials. Part II, Ann. Inst. Fourier, Grenoble 17, 2 (1967), 1-135. MR 37:4281

[2] C. Bennett, R. DeVore and R. Sharpley, Weak- $L^{\infty}$ and BMO, Ann. of Math. 113 (1981), 601-611. MR 82h:46047

[3] C. Bennett and R. Sharpley, Interpolation of Operators, Academic Press, 1988. MR 89e: 46001

[4] V. I. Burenkov and W. D. Evans, Weighted Hardy-type inequalities for differences and the extension problem for spaces with generalized smoothness, J. London Math. Soc. 57 (1998), 209-230. MR 99c:46025

[5] P. Grisvard, Espaces intermédiaires entre espaces de Sobolev avec poids, Ann. Scuola Norm. Sup. Pisa 17 (1963), 255-296.

[6] H. P. Heinig, A. Kufner and L. E. Persson, On some fractional order Hardy inequalities, J. Inequal. Appl. 1 (1997), 25-46.

[7] N. Krugljak, L. Maligranda and L. E. Persson, The failure of the Hardy inequality and interpolation of intersections, Arkiv Mat., to appear.

[8] A. Kufner and H. Triebel, Generalizations of Hardy's inequality, Conf. Sem. Mat. Univ. Bari 156 (1978), 1-21. MR 81a:26014

[9] J. L. Lions and E. Magenes, Problémes aux Limites Non Homogénes et Applications I, Springer-Verlag, Berlin-New York, 1972.

[10] J. L. Lions and E. Magenes, Problémes aux limites non homogénes IV, Scuola Norm. Sup. Pisa 15 (1961), 311-326.

[11] M. Milman and Y. Sagher, An interpolation theorem, Arkiv Mat. 22 (1984), 33-38. MR 85k:46084b

[12] B. Opic and A. Kufner, Hardy-Type Inequalities, Longman Scientific \& Technical, Harlow, 1990. MR 92b:26028

[13] G. N. Yakovlev, Boundary properties of functions from the space $W_{p}^{(1)}$ on domains with angular points, Dokl. Akad. Nauk. SSSR 140 (1961), 73-76. (Russian)

Department of Mathematics, Yaroslavl State University, Sovetskaya 14, 150000 YAROSLAVL, Russia

E-mail address: natan@univ.uniyar.ac.ru

Department of Mathematics, Lule̊̊ University of Technology, S-971 87 Lule̊, SweDEN

E-mail address: lech@sm.luth.se

E-mail address: larserik@sm.luth.se 